\title{
ON BITOPOLOGICAL QUASI-PSEUDOMETRIZATION
}

\author{
S. ROMAGUERA \\ (Received 6 November 1981; revised 10 October 1982) \\ Communicated by J. H. Rubinstein
}

\begin{abstract}
In this paper we give a sufficient condition of quasi-pseudometrization for bitopological spaces. From this condition we obtain, as immediate corollaries, some known results.
\end{abstract}

1980 Mathematics subject classification (Amer. Math. Soc.): primary 54 E 55; secondary 54 E 35.

Keywords and phrases: Bitopological spaces, quasi-pseudometrization.

\section{Introduction}

A quasi-pseudometric space is a pair $(X, d)$ where $X$ is a set and $d$ is a mapping from $X \times X$ into the real numbers $\mathbf{R}$ satisfying for all $x, y, z \in X$ : (i) $d(x, y) \geqslant 0$, (ii) $d(x, x)=0$, (iii) $d(x, y) \leqslant d(x, z)+d(z, y)$. From a quasi-pseudometric, we can determine two topologies on $X$ in a natural way: $\tau_{d}=\{A \subset X: \forall x \in A$ there is a $r>0$ such that $\left.B_{d}(x, r) \subset A\right\}$ with $B_{d}(x, r)=\{y \in X: d(x, y)<r\}$ and $\tau^{d}=\left\{A \subset X: \forall x \in A\right.$ there is a $r>0$ such that $\left.B^{d}(x, r) \subset A\right\}$ with $B^{d}(x, r)=$ $\{y \in X: d(y, x)<r\}$.

Kelly [2] began the study of bitopological spaces and its quasi-pseudometrizability. Also Patty [4], Lane [3] and Salbany [5] have contributions. Salbany gives an interesting sufficient condition for quasi-pseudometrizability from which we deduce a generalization of the Nagata-Smirnov theorem, solving with it a conjecture of Patty. Here we obtain (Theorem 1) another sufficient condition of quasi-pseudometrization from which we deduce (Corollary 1.1) that is $(X, \mathscr{P}, \mathcal{Q})$ is a pairwise perfectly normal space, $\mathscr{P}$ has a $\mathcal{Q}-\sigma$-locally finite base and $\mathcal{L}$ has a

(c) 1984 Australian Mathematical Society 0263-6115/84 \$A2.00+0.00 
$\mathscr{P}$-o-locally finite base, then $(X, \mathscr{P}, \mathscr{Q})$ is quasi-pseudometrizable. This result modifies an incorrect assertion of Salbany (Example 1). Several standard theorems of the theory of quasi-pseudometrizability are easily deduced from Corollary 1.1 .

\section{Results}

THEOREM 1. Let $(X, \mathcal{P}, \mathcal{Q})$ be a bitopological space such that $\mathscr{P}$ has a 2 - -locally finite base $\mathfrak{Q}=\cup_{n=1}^{\infty} \mathbb{Q}_{n}$ and 2 has a $\mathscr{P}$ - $\sigma$-locally finite base $\mathscr{G}=\cup_{n=1}^{\infty} \mathscr{B}_{n}$. Then, $(X, \mathscr{P}, \mathcal{2})$ is quasi-pseudometrizable if, and only if, we verify:

(a) For every $A \in \mathbb{Q}$ there is a function $f_{A}: X \rightarrow[0,1]$ Q-upper semi-continuous such that $f_{A}^{-1}(0)=X-A$.

(b) For every $j$ and every sequence $\left\{A_{n}\right\}_{n=1}^{\infty} \subset Q_{j}$, if $\mathcal{P}-\lim _{n} x_{n}=x$ then $\lim _{n} \max \left[f_{A_{n}}(x)-f_{A_{n}}\left(x_{n}\right), 0\right]=0$.

(a') For every $B \in \mathscr{B}$ there is a function $g_{B}: X \rightarrow[0,1] \mathscr{P}$-upper semi-continuous such that $g_{B}^{-1}(0)=X-B$.

$\left(b^{\prime}\right)$ For every $j$ and every sequence $\left\{B_{n}\right\}_{n=1}^{\infty} \subset \mathscr{B}_{j}$, if $\mathcal{L}-\lim _{n} x_{n}=x$ then $\lim _{n} \max \left[g_{B_{n}}(x)-g_{B_{n}}\left(x_{n}\right), 0\right]=0$.

Proof. For each couple of points $x, y \in X$ and each $n \in \mathbf{N}$ we can define $d_{n}(x, y)=\sup _{A \in \mathbb{Q}_{n}}\left\{\max \left[f_{A}(x)-f_{A}(y), 0\right]\right\}$ and $d(x, y)=\sum_{n=1}^{\infty} 2^{-n} d_{n}(x, y)$.

The mapping $d$ is a quasi-pseudometric for $X$. We have to prove that $\tau_{d}=\mathscr{P}$ and $\tau^{d} \subset \mathcal{Q}$.

To verify the first equality we use a technique of Borges [1]: If $\lim _{n} d\left(x, x_{n}\right)=0$ but $\mathcal{P}-\lim _{n} x_{n} \neq x$ then there exists $A \in$ some $\mathcal{Q}_{m}$ which is a neighbourhood of $x$ and a subsequence $\left\{x_{n_{k}}\right\}_{k=1}^{\infty}$ of $\left\{x_{n}\right\}_{n=1}^{\infty}$ such that $A \cap\left\{x_{n_{k}}\right\}_{k=1}^{\infty}=\varnothing$. Then $f_{A}\left(x_{n_{k}}\right)=0$ for every $k$, and $f_{A}(x) \neq 0$, therefore $\lim _{k} d\left(x, x_{n_{k}}\right) \neq 0$. Now, if $\mathscr{P}-\lim _{n} x_{n}=x$, for every $\varepsilon>0$ there is an $m \in \mathbf{N}$ such that $2^{-m+2}<\varepsilon$; then $\varepsilon / 2>\sum_{r=m}^{\infty} 2^{-r} d_{r}\left(x, x_{n}\right)$ for every $x_{n}$. If $r<m, \lim _{n} d_{r}\left(x, x_{n}\right)=0$ : otherwise, there exists $p<m$ such that $\lim _{n} d_{p}\left(x, x_{n}\right) \neq 0$; so we can find $\delta>0$ and a subsequence $\left\{x_{n_{k}}\right\}_{k=1}^{\infty}$ of $\left\{x_{n}\right\}_{n=1}^{\infty}$ such that $d_{p}\left(x, x_{n_{k}}\right) \geqslant \delta$ for every $k$. Consequently, there is an $A_{k} \in \mathbb{Q}_{p}$ for every $k$, with $\max \left[f_{A_{k}}(x)-f_{A_{k}}\left(x_{k}\right), 0\right]>\delta / 2$; then we have $\lim _{k} \max \left[f_{A_{k}}(x)-f_{A_{k}}\left(x_{k}\right), 0\right] \geqslant \delta$ which is a contradiction with (b). Then $\mathscr{P}=\tau_{d}$.

To prove $\tau^{d} \subset \mathscr{Q}$ it suffices to show that, for every $x \in X$ and $\varepsilon>0,\{y \in X$ : $d(y, x)<\varepsilon\}$ is a 2-open set. For a fixed $x_{0} \in X$, let $x \in X$ and $\varepsilon>0$, then there is a 2 -neighbourhood $W$ of $x$ with $W \cap A_{i} \neq \varnothing, i=1,2, \ldots, p$, being $A_{i} \in \mathbb{Q}_{n}$, and $W \cap A=\varnothing$ for every $A \in \mathbb{Q}_{n}-\left\{A_{1}, A_{2}, \ldots, A_{p}\right\}$. Since $f_{A_{i}}$ is 2-upper 
semi-continuous, $i=1,2, \ldots, p$, there is a 2 neighbourhood $V_{i}$ of $x$ with $f_{A_{i}}(x)-$ $f_{A}(y)<\varepsilon$ for every $y \in V_{i}$. Let $V=W \cap\left(\cap_{i=1}^{p} V_{i}\right) ; V$ is a 2 neighbourhood of $x$ and for every $y \in V$ we get that:

$$
\begin{aligned}
d_{n}\left(y, x_{0}\right)-d_{n}\left(x, x_{0}\right) & \leqslant d_{n}(y, x)=\sup _{A \in \mathbb{Q}_{n}}\left\{\max \left[f_{A}(y)-f_{A}(x), 0\right]\right\} \\
& =\max \left\{\max \left[f_{A_{i}}(y)-f_{A_{i}}(x), 0\right], i=1,2, \ldots, p\right\}<\varepsilon .
\end{aligned}
$$

Then $\tau^{d} \subset \mathscr{Q}$.

Similarly, we can define for every $x, y \in X$ and every $n \in \mathbf{N} d_{n}^{\prime}(x, y)=$ $\sup _{B \in \mathscr{B}_{n}}\left\{\max \left[g_{B}(x)-g_{B}(y), 0\right]\right\}$ and $d^{\prime}(x, y)=\sum_{n=1}^{\infty} 2^{-n} d_{n}^{\prime}(x, y), d^{\prime}$ is a quasipseudometric for $X$ with $\tau_{d^{\prime}}=2$ and $\tau^{d^{\prime}} \subset \mathcal{P}$. Then, the quasi-pseudometric $d^{\prime \prime}(x, y)=d^{\prime}(y, x)$ verifies $\tau^{d^{\prime \prime}}=2$ and $\tau_{d^{\prime \prime}} \subset \mathscr{P}$. Now, let $\bar{d}(x, y)=d(x, y)+$ $d^{\prime \prime}(x, y)$. This quasi-pseudometric for $X$ verifies $\mathcal{P}=\tau_{\bar{d}}^{-}$and $\mathscr{L}=\tau^{\bar{d}}$.

Conversely, let $(X, \mathscr{P}, \mathscr{2})$ be a quasi-pseudometrizable bitopological space. There is a quasi-pseudometric $d$ such that $\tau_{d}=\mathscr{P}$ and $\tau^{d}=\mathcal{L}$, we can suppose that $d$ has diameter lower than 1 . Let $A$ be a $\mathcal{P}$-open set in $X$ and $f_{A}(x)=$ $d(x, X-A)$. Obviously, $f_{A}$ verifies (a) and (b). In the same way we verify $\left(a^{\prime}\right)$ and $\left(b^{\prime}\right)$. The proof is complete.

The above result is a weak bitopological version of Borges' topological theorem [1], page 801 , since we only obtain a sufficient condition for quasi-pseudometrization.

COROLlaRY 1.1. If $(X, \mathcal{P}, \mathcal{Q})$ is a pairwise perfectly normal space, $\mathcal{P}$ has a Q- $\sigma$-locally finite base $\mathcal{Q}=\cup_{n=1}^{\infty} \mathbb{Q}_{n}$ and $\mathcal{Q}$ has a $\mathcal{P}$ - $\sigma$-locally finite base $\mathfrak{B}=$ $\cup_{n=1}^{\infty} \mathscr{G}_{n}$, then $(X, \mathscr{P}, \mathcal{Q})$ is quasi-pseudometrizable.

Proof. For each $A \in Q$, there is by [3] a $\mathscr{P}$-lower semi-continuous and $\mathscr{2}$-upper semi-continuous function $f_{A}: X \rightarrow[0,1]$ such that $f_{A}^{-1}(0)=X-A$. By the 2-local finiteness of every $\mathfrak{Q}_{j}$ condition (b) of Theorem 1 follows. Likewise are verified conditions $\left(a^{\prime}\right)$ and $\left(b^{\prime}\right)$. By Theorem $1,(X, \mathscr{P}, \mathcal{L})$ is quasi-pseudometrizable.

Corollary 1.2 (Salbany). If $(X, \mathscr{P}, \mathcal{Q})$ is a pairwise regular space, $\mathscr{P}$ has a

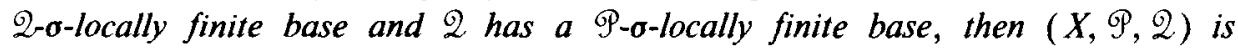
quasi-pseudometrizable.

Proof. By $[4](X, \mathscr{P}, \mathcal{2})$ is pairwise perfectly normal and by Corollary 1.1 we have the result. 
Note. This corollary is also obtained by Salbany as corollary of another more general result.

Corollary 1.3 (Kelly). If ( $X, \mathcal{P}, \mathcal{Q})$ is a pairwise regular space and $\mathscr{P}$ and $\mathscr{Q}$ have countable basis, then $(X, \Im, \mathcal{Q})$ is quasi-pseudometrizable.

Salbany asserts, [5], page 302, that if $(X, \mathscr{P}, \mathcal{Q})$ is pairwise normal and $\mathscr{P}$ has a Q $\sigma$-locally finite base, then $\mathscr{P}=\tau_{d}$ and $\mathcal{L} \supset \tau^{d}$ for some quasi-pseudometric $d$. The following example proves that this result is not correct.

EXAmple 1. Let $(X, \mathscr{P}, 2)$ be such that $X=\mathbf{R}$ (the set of reals), $\mathscr{P}$ is the euclidean topology and 2 is the trivial topology. $(X, \mathscr{P}, \mathcal{2})$ is pairwise normal and if $d$ is a quasi-pseudometric such that $\mathscr{P}=\tau_{d}$ and $\mathcal{Q} \supset \tau^{d}$, then $\mathcal{Q}=\tau^{d}$ which is a contradiction since $(X, \mathscr{P}, \mathscr{Q})$ is not pairwise regular.

\section{Acknowledgement}

The author is grateful to Professor Manuel López Pellicer for his assistance with the writing of this paper.

\section{References}

[I] C. J. Borges, 'On metrizability of topological spaces', Canad. J. Math. 20 (1968), 795-804.

[2] J. C. Kelly, 'Bitopological spaces', Proc. London Math. Soc. 13 (1963), 71-89.

[3] E. P. Lane, 'Bitopological spaces and quasi-uniform spaces', Proc. London Math. Soc. 17 (1967), $241-256$.

[4] C. W. Patty, "Bitopological spaces', Duke Math. J. 34 (1967), 387-392.

[5] S. Salbany, 'Quasi-metrization of bitopological spaces', Arch. Math. (Basel) 23 (1972), 299-306.

Departmento de Matemáticas II

ETSICCP

Universidad Politécnica

Valencia-22

Spain 